\title{
Tromboembolia arterial y amputación de una extremidad en un neonato posterior al síndrome de dificultad respiratoria. Caso clínico
}

\author{
Neonatal arterial thromboembolism and limb loss following respiratory \\ distress syndrome. Case report
}

Dra. Sung Shin Kima ${ }^{a}$ Dr. Ihl Sung Park ${ }^{b}$ y Dra. Hyun Sook Hong ${ }^{c}$

\section{RESUMEN}

La tromboembolia en los recién nacidos es un problema grave. Los factores de riesgo más importantes son iatrogénicos, entre ellos, el uso de sondas umbilicales o vías centrales permanentes. Entre otros factores de riesgo, se incluyen la asfixia, la deshidratación, la septicemia, la cardiopatía, el síndrome de dificultad respiratoria, la coagulación intravascular diseminada, la trombofilia congénita (deficiencia de proteína $C$ o proteína S), la diabetes mellitus materna y la transferencia pasiva de anticuerpos antifosfolípidos maternos. Los recién nacidos son más vulnerables que los adultos a presentar una trombosis debido a su menor capacidad fibrinolítica.

Describimos el caso de una recién nacida de un día de vida con tromboembolia arterial en el miembro inferior, sin vía central. La tromboembolia en este caso estuvo asociada con un síndrome de dificultad respiratoria. Se realizó trombólisis, tratamiento anticoagulante y trombectomía quirúrgica. Posteriormente, se amputó el miembro inferior por debajo de la rodilla.

Palabras clave: amputación, arterias, sindrome de dificultad respiratoria del recién nacido, tromboembolia

http:/ /dx.doi.org/10.5546/aap.2015.e157

\section{INTRODUCCIÓN}

La tromboembolia es una enfermedad rara pero grave en los recién nacidos que exige cuidados intensivos. Las consecuencias pueden ser devastadoras si no se realiza un tratamiento oportuno y preciso. Varios factores contribuyen a un mayor riesgo de tromboembolia, por ejemplo,

a. Departamento de Pediatría, Facultad de Medicina, Universidad de Soonchunhyang, Hospital de Bucheon, República de Corea.

b. Departamento de Pediatría, Facultad de Medicina, Universidad de Soonchunhyang, Hospital de Cheonan, República de Corea.

c. Departamento de Radiología, Facultad de Medicina, Universidad de Soonchunhyang, Hospital de Bucheon, República de Corea.

Correspondencia:

Dra. Sung Shin Kim: sungshin1201@gmail.com

Financiamiento: este trabajo ha sido parcialmente financiado por Soonchunhyang University Research Fund.

Conflicto de intereses: Ninguno que declarar.

Recibido: 9-10-2014

Aceptado: 3-12-2014 la colocación de sondas venosas y arteriales, ${ }^{1}$ las cardiopatías, ${ }^{2}$ la diabetes materna, ${ }^{3,4}$ la transferencia de anticuerpos antifosfolípidos, ${ }^{5}$ la deshidratación, la septicemia, el síndrome de dificultad respiratoria (SDR), la coagulación intravascular diseminada y la asfixia. ${ }^{4}$ Presentamos el caso de una recién nacida con tromboembolia arterial sometida a una amputación después de presentar SDR.

\section{PRESENTACIÓN DEL CASO}

Recién nacida mediante cesárea a las 36 semanas de gestación, con 3100 g de peso, de una madre de 30 años, grávida 2, para 1. Los resultados de las pruebas de pesquisa prenatal en suero materno no presentaron particularidades. No tenía antecedentes de hipertensión, lupus eritematoso sistémico, diabetes mellitus, nefropatía ni diátesis trombótica. A las 4 horas de vida, la recién nacida presentó cianosis y taquipnea. Se la trasladó al hospital más cercano. La radiografía de tórax mostró opacidades en vidrio esmerilado en ambos pulmones con márgenes cardíacos mal definidos. Se la conectó a un respirador y se le administró surfactante. En este momento, no tenía una vía central. A las 12 horas de vida, la pierna derecha estaba pálida y fría, $3 \mathrm{~cm}$ por debajo de la rodilla (Figura 1). No se palpaba el pulso

FIGURA 1. Isquemia que compromete la pierna derecha

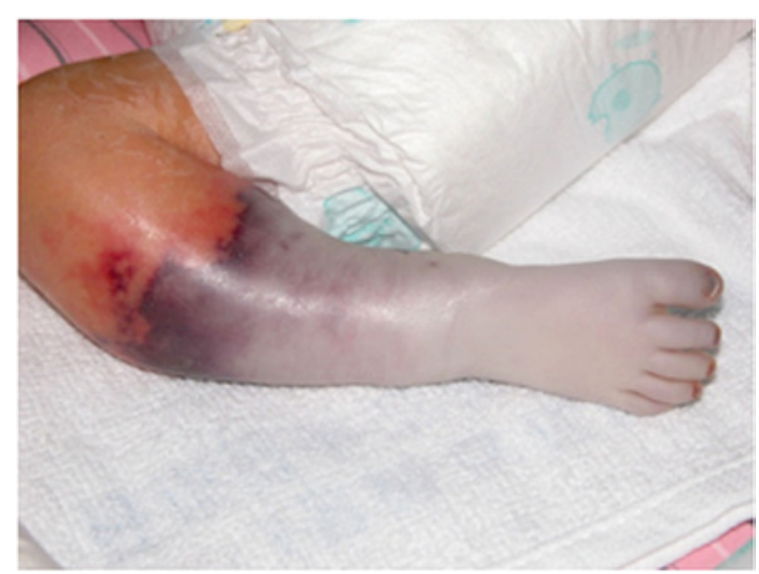


de la arteria dorsal del pie derecho. El recuento de leucocitos fue de $16370 / \mathrm{mm}^{3}$ con $75 \%$ de neutrófilos. Los niveles de hematocrito y proteína $C$ reactiva (PCR) fueron normales. Se evaluó la posibilidad de trombofilia. Los niveles de proteína C, proteína S y antitrombina fueron normales. No se hallaron polimorfismo C677T homocigoto del gen metilentetrahidrofolato reductasa (MTHFR), factor de $\mathrm{V}$ Leiden heterocigoto, ni mutación G20210A del gen de la protrombina. El recuento de plaquetas fue de $96000 / \mathrm{mm}^{3}$. El tiempo de protrombina fue de 15,6 s (normal, 10,1-15,9 s). El tiempo de tromboplastina parcial activada fue de 40,9 s (normal, 31,3-54,3 s). La concentración de fibrinógenos fue de $115 \mu \mathrm{g} / \mathrm{dl}$ (normal, 167$399 \mu \mathrm{g} / \mathrm{dl}$ ). La concentración del dímero D fue de $13536 \mu \mathrm{g} / \mathrm{L}$ (normal, 0-500 $\mathrm{\mu g} / \mathrm{L}$ ). En la imagen reconstruida de la angiografía por tomografía computada (TC) no se observó la arteria ilíaca interna que deriva de la bifurcación ilíaca (Figura 2). Sin embargo, se visualizó la arteria femoral derecha a la altura de la mitad del muslo mediante recanalización. A partir de allí, tampoco se observaron las arterias distales, poplíteas, tibiales

FIGURA 2. Angiografía por tomografía computada (TC) en la que no se visualiza la arteria ilíaca interna derecha que deriva de la bifurcación ilíaca. Sin embargo, se visualiza la arteria femoral derecha a la altura de la mitad del muslo después de una recanalización. No se observan las arterias distales, popliteas, ni las tibiales anterior y posterior

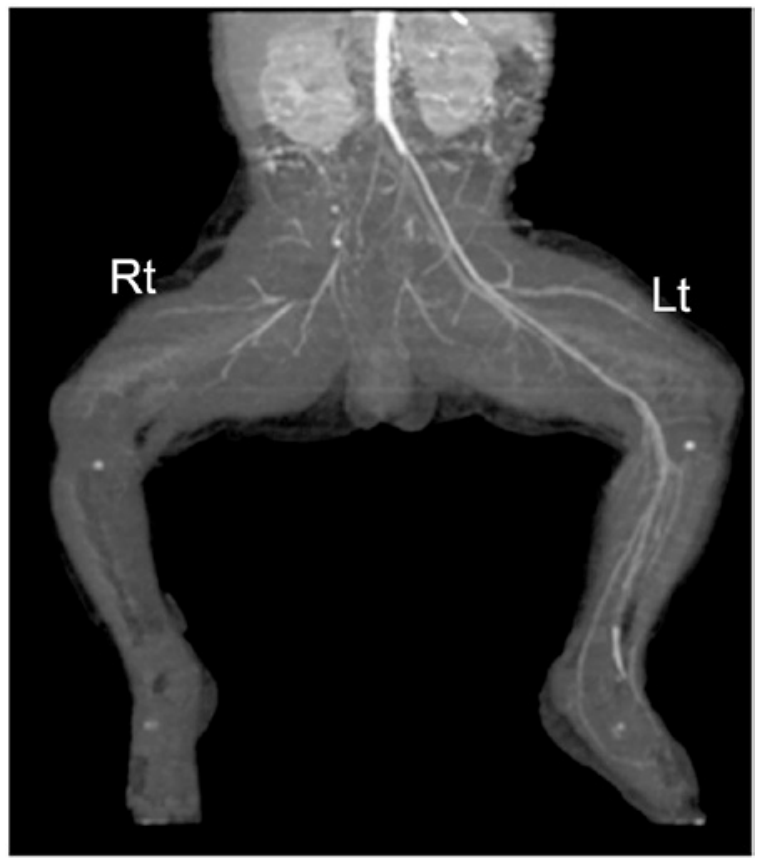

anterior y posterior. El pico del flujo de la arteria femoral derecha era bajo y más lento que el de la arteria femoral izquierda. El flujo venoso estaba conservado (Figura 3). Tras detectar la oclusión vascular causada por la tromboembolia arterial, de inmediato se realizó trombólisis y se administró activador del plasminógeno tisular (APT) en dosis de $0,5 \mathrm{mg} / \mathrm{kg} / \mathrm{h}$ durante 6 horas y plasma congelado en fresco. Simultáneamente, se administró heparina no fraccionada $(20 \mathrm{U} / \mathrm{kg} / \mathrm{h})$ para mantener la anticoagulación en dosis bajas.

Antes del tratamiento trombolítico, se realizó una ecografía cerebral para investigar hemorragias. A pesar del tratamiento, la viabilidad de la pierna no mejoró. Al tercer día se realizó una trombectomía quirúrgica. No fue posible restaurar el flujo arterial de la pierna. A los 20 días de vida, se realizó una amputación por debajo de la rodilla.

\section{DISCUSIÓN}

Los lactantes presentan riesgo de tromboembolia debido a factores genéticos y adquiridos. ${ }^{6}$ El estado hipofibrinolítico (caracterizado por una respuesta anticoagulatoria menos desarrollada con deficiencia de antitrombina, proteína $\mathrm{C}$ y proteína $S$ ) aumenta el riesgo de trombosis en los recién nacidos hasta restaurar el equilibrio. Los defectos trombofílicos heredados, como polimorfismos protrombóticos, factor V G1691A, factor II G2021A y genotipo TT homocigoto del polimorfismo C677T del gen metilentetrahidrofolato reductasa, también aumentan el riesgo de trombosis. ${ }^{7}$ En el caso de una tromboembolia, se recomienda investigar la posibilidad de trombofilia heredada para la orientación familiar, y el tratamiento y control a largo plazo. El riesgo puede estar exacerbado por otros factores, como la diabetes mellitus, la policitemia, la septicemia, la cardiopatía, la asfixia y el SDR. ${ }^{8}$ Los recién nacidos críticamente enfermos son más susceptibles a tener tromboembolia que los recién nacidos sanos. ${ }^{9}$ Los lactantes son más susceptibles a presentar tromboembolia arterial en los miembros inferiores. Los niños mayores tienen más probabilidades de desarrollar tromboembolia en el sistema nervioso central. ${ }^{10}$ Según el Registro Internacional Canadiense, el 89\% de los casos de trombosis estuvieron asociados con la colocación de catéteres intravasculares. ${ }^{11}$

Brus, et al. ${ }^{12}$ informaron que la formación de coágulos, la fibrinólisis y el sistema calicreínacininas se activan simultáneamente en los recién 
FIGURA 3. Ecografía Doppler de la pierna que muestra que el pico del flujo de la arteria femoral derecha es bajo y lento en comparación con el flujo amplio de la arteria femoral izquierda. El flujo venoso está conservado. La definición del flujo de la arteria poplitea derecha es deficiente (no se muestra). (A) Arterial femoral derecha, (B) Arterial femoral izquierda, (C) Vena femoral derecha

A.

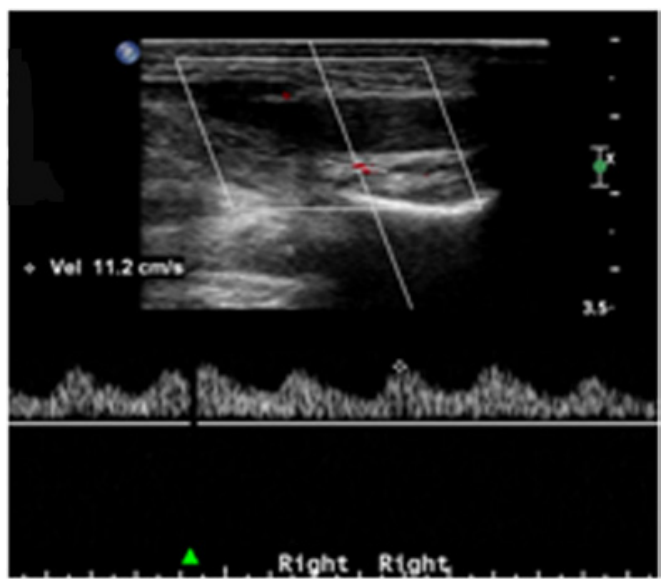

B.

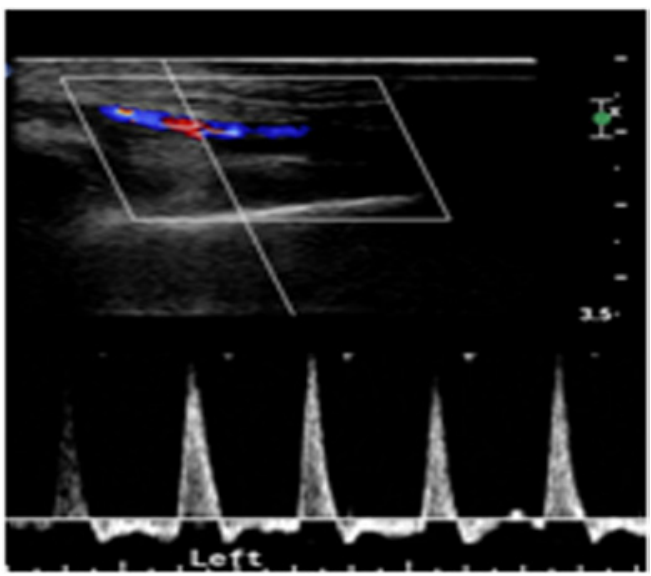

C.

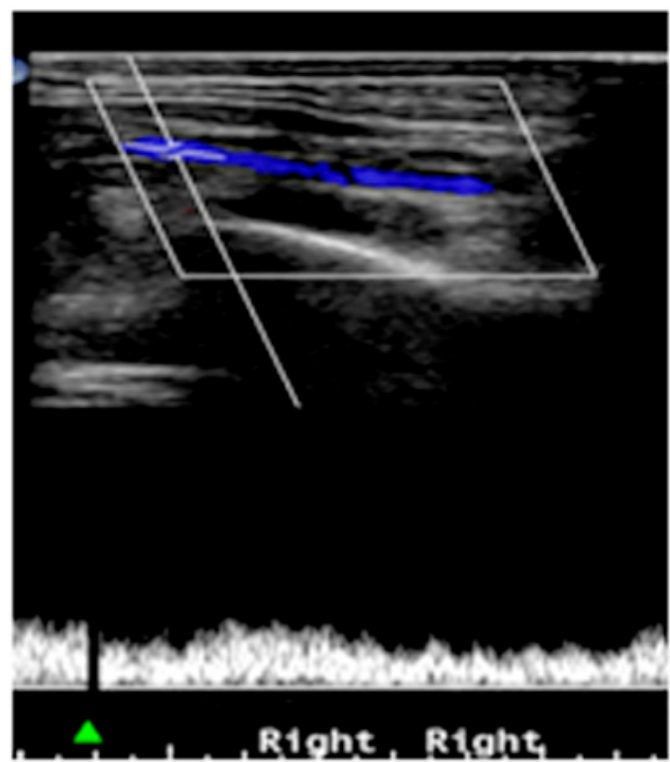

nacidos prematuros con SDR grave, lo que resulta en alteraciones en la coagulación. La actividad fibrinolítica puede provocar tromboembolia. El diagnóstico se puede realizar mediante una ecografía Doppler, una TC, una resonancia magnética (RM) con o sin angiografía por resonancia magnética y una venografía. La ecografía Doppler es la técnica utilizada más frecuentemente para confirmar tromboembolia en los recién nacidos críticamente enfermos.

La posibilidad de salvar la extremidad depende del tiempo transcurrido entre el evento vascular inicial, la presentación y el tratamiento. ${ }^{8}$ Entre los tratamientos complementarios se incluyen la hidratación, los antibióticos, los vasodilatadores sistémicos o tópicos, la oxigenoterapia hiperbárica y la anticoagulación sistémica. ${ }^{13}$ En comparación con los adultos, la tromboembolia es rara en los niños, lo que dificulta la realización de estudios clínicos. Para la anticoagulación, deben usarse heparina de bajo peso molecular o heparina no fraccionada. La duración total de la anticoagulación es entre seis semanas y tres meses. ${ }^{14}$

El tratamiento trombolítico está indicado solamente cuando la oclusión de los grandes vasos compromete críticamente los órganos o las extremidades, o cuando no se observan cambios fijos en la piel con necrosis establecida, o cuando la oclusión es breve pero se presenta con isquemia. ${ }^{8} \mathrm{Si}$ es necesario realizar una trombólisis, se recomienda utilizar un activador del plasminógeno tisular y suplementación con plasminógeno. La heparina debe coadministrarse en dosis profilácticas. ${ }^{15}$ Puede producirse hemorragia intracraneal en hasta el 10\% de los recién nacidos prematuros que reciben la dosis más alta de $\mathrm{APT}{ }^{15}$

Entre las contraindicaciones de la trombólisis se incluyen la cirugía o hemorragia mayor, la asfixia grave, un procedimiento invasivo, las convulsiones, el haber nacido con menos de 32 semanas de gestación, la septicemia, la incapacidad para mantener la concentración de plaquetas $>50-100000 / \mu l$ y la concentración de fibrinógenos $>100 \mathrm{mg} / \mathrm{dl} .{ }^{18}$ Se recomienda la intervención quirúrgica cuando el tratamiento trombolítico está contraindicado y existe la posibilidad de necrosis de un órgano o de una extremidad. ${ }^{14} \mathrm{La}$ amputación debe retrasarse siempre que sea posible ya que la línea de demarcación podría estar muy alejada de la línea original de la isquemia. La amputación debe realizarse solamente después de considerar la 
colocación de una extremidad protésica en el futuro y la prevención de la contractura de la articulación. ${ }^{8}$

Si se observa trombosis en un paciente críticamente enfermo, con o sin factores de riesgo, se lo debe manejar rápida y cuidadosamente para salvar el órgano o prevenir la pérdida de la extremidad. Hasta donde sabemos, esta es la primera presentación de un caso de tromboembolia arterial que condujo a la amputación de la pierna después de un SDR sin el uso de una vía central.

\section{Reconocimientos}

Este trabajo estuvo parcialmente respaldado por el Fondo de Investigación de la Universidad Soonchunhyang.

\section{REFERENCIAS}

1. Boo NY, Wong NC, Zulkifli SS, Lye MS. Risk factors associated with umbilical vascular catheter-associated thrombosis in newborn infants. J Paediatr Child Health 1999;35(5):460-5.

2. Sträter R, Vielhaber H, Kassenböhmer R, von Kries R, et al. Genetic risk factors of thrombophilia in ischaemic childhood stroke of cardiac origin. A prospective ESPED survey. Eur J Pediatr 1999;158 Suppl 3:S122-5.

3. Moazzam A, Riaz M, Brennen MD. Neonatal gangrene in an extremity of an infant of a diabetic mother. BJOG 2003;110(1):74-6.

4. Nowak-Göttl U, Kosch A, Schlegel N. Neonatal thromboembolism. Semin Thromb Hemost 2003;29(2):227-34.
5. Bhat MA, Kawoosa MS, Bhat JI, Ali SW. Antiphospholipid syndrome in a neonate. Pediatr Dermatol 2011;28(3):342-5.

6. Thornburg C, Pipe S. Neonatal thromboembolic emergencies. Semin Fetal Neonatal Med 2006;11(3):198-206.

7. Veldman A, Nold MF, Michel-Behnke I. Thrombosis in the critically ill neonate: incidence, diagnosis, and management. Vasc Health Risk Manag 2008;4(6):1337-48.

8. Arshad A, McCarthy MJ. Management of limb ischaemia in the neonate and infant. Eur J Vasc Endovasc Surg 2009;38(1):61-5.

9. Beardsley DS. Venous thromboembolism in the neonatal period. Semin Perinatol 2007;31(4):250-3.

10. Monagle P, Newall F, Barnes C, Savoia H, et al. Arterial thromboembolic disease: a single-centre case series study. J Paediatr Child Health 2008;44(1-2):28-32.

11. Schmidt B, Andrew M. Neonatal thrombosis: report of a prospective Canadian and international registry. Pediatrics 1995;96(5 Pt 1):939-43.

12. Brus F, Van Oeveren W, Okken A, Oetomo SB. Disease severity is correlated with plasma clotting and fibrinolytic and kinin-kallikrein activity in neonatal respiratory distress syndrome. Pediatr Res 1997;41(1):120-7.

13. Wiebers J, Purdy I, Lieber M, Milisavljevic V. Hyperbaric oxygen in treatment of neonatal arterial thromboembolism of lower extremities. J Perinatol 2006;26(12):777-9.

14. Monagle P, Chalmers E, Chan A, DeVeber G, et al. Antithrombotic therapy in neonates and children: American College of Chest Physicians Evidence-Based Clinical Practice Guidelines (8th Edition). Chest 2008;133(6 Suppl):887s-968s.

15. Manco-Johnson MJ, Grabowski EF, Hellgreen M, Kemahli AS, et al. Recommendations for tPA thrombolysis in children. On behalf of the Scientific Subcommittee on Perinatal and Pediatric Thrombosis of the Scientific and Standardization Committee of the International Society of Thrombosis and Haemostasis. Thromb Haemost 2002;88(1):157-8. 